\title{
Transcriptional changes induced by epigenetic therapy with hydralazine and magnesium valproate in cervical carcinoma
}

\author{
ERICK DE LA CRUZ-HERNÁNDEZ ${ }^{1}$, CARLOS PEREZ-PLASENCIA ${ }^{1}$, ENRIQUE PÉREZ-CARDENAS ${ }^{1}$, \\ AURORA GONZALEZ-FIERRO ${ }^{1}$, CATALINA TREJO-BECERRIL ${ }^{1}$, ALMA CHÁVEZ-BLANCO ${ }^{1}$, \\ LUCIA TAJA-CHAYEB ${ }^{1}$, SILVIA VIDAL ${ }^{1}$, OLGA GUTIÉRREZ ${ }^{1}$, GUADALUPE I. DOMINGUEZ ${ }^{1}$, \\ JAENAI E. TRUJILLO ${ }^{1}$ and ALFONSO DUENAS-GONZÁLEZ ${ }^{2}$ \\ ${ }^{1}$ Unidad de Investigación Biomédica en Cáncer, Instituto de Investigaciones Biomédicas (IIB), Universidad Nacional \\ Autonóma de Mexico (UNAM), Instituto Nacional de Cancerología (INCan), Mexico City; \\ ${ }^{2}$ Division of Basic Research, Instituto Nacional de Cancerologia, Mexico City, Mexico
}

Received July 1, 2010; Accepted September 29, 2010

DOI: $10.3892 /$ or.2010.1086

\begin{abstract}
Aberrant DNA methylation and histone deacetylation participate in cancer development and progression; hence, their reversal by inhibitors of DNA methylation and histone deacetylases is a promising cancer therapy. Experimental data demonstrate that these inhibitors in combination do not only show synergy in antitumor effects but also in whole genome global expression. Ten pairs of pre- and posttreatment cervical tumor samples were analyzed by microarray analysis. Treatment for seven days with hydralazine and valproate (HV) in patients up-regulated 964 genes. The two pathways possessing the highest number of up-regulated genes comprised the ribosome protein and the oxidative phosphorylation pathways, followed by MAPK signaling, tight junction, adherens junction, actin cytoskeleton, cell cycle, focal adhesion, apoptosis, proteasome, Wnt signaling, and antigen processing and presentation pathways. Upregulated genes by $\mathrm{HV}$, clustered with down-regulated genes in untreated primary cervical carcinomas and were more alike as compared with up-regulated genes from untreated patients in terms of gene ontology. Increased acetylated p53 was also observed. Epigenetic therapy with HV leads to gene reactivation in primary tumors of cervical cancer patients as well as protein acetylation. A number of these reactivated genes have a definitive role as a tumor suppressors. The global expression pattern induced by HV suggests this therapy has an impact on pathways related to energy production which may promote apoptosis.
\end{abstract}

Correspondence to: Dr Alfonso Duenas-González, Instituto Nacional de Cancerología, Av. San Fernando 22, Tlalpan, 14080 México, D.F., México

E-mail: alfonso_duenasg@yahoo.com

Key words: epigenetic therapy, hydralazine, valproate, cervical cancer, microarray

\section{Introduction}

DNA methylation (DNMT) and histone deacetylase inhibitors (HDAC) exert their antitumor effects by inhibiting cell proliferation, metastases, angiogenesis, and by inducing cell differentiation and/or apoptosis, as well as by increasing chemotherapy cytotoxicity (1-5). Further, these epigenetic drugs are radiosensitizers (6-8).

The rationale for utilizing these epigenetic drugs for cancer treatment has relied on the theory that reversing epigenetic aberrations would turn-on tumor suppressor genes and consequently exert antitumor effects. Most of this knowledge however, has been gained in experimental systems by candidate-gene approach which is a biased method. On the contrary, epigenomic pharmacological reactivation strategy in either cancer cell lines or cancer patients followed by global gene expression analysis may identify up-regulated genes in an unbiased manner (9).

Synergy between DNMT and HDAC inhibitors on gene re-expression at a global scale is already known (10) indicating that the reversal of these two epigenetic factors synergizes gene re-expression (11). We have shown that in the SW480 colon cancer cell line, the combination of hydralazine and valproic acid has a synergistic effect on gene reactivation (5).

We recently completed a proof-of-principle study of epigenetic therapy (12) with the DNA methylation and HDAC inhibitors hydralazine and valproate (HV) respectively, added to neoadjuvant doxorubicin cyclophosphamide for locally advanced breast cancer in which we demonstrate global DNA hypomethylation and HDAC activity inhibition in the peripheral blood of patients, as well as gene up-regulation of $>1,000$ genes in primary breast tumors after 7 days of treatment with HV (12). However, because of the technical difficulties for taking post-treatment tumor samples from the breast, these data emerged from the analysis of only a pair of pre- and post-treatment samples and 2 additional pre-treat-ment samples. Here, we extended those observations in a trial performed in cervical cancer patients. 


\section{Patients and methods}

Primary cervical cancer samples. Punch biopsies were taken from primary cervical tumors at diagnosis and at day 8 of treatment with hydralazine and magnesium valproate prior to first dose of cisplatin and external radiation. Part of the biopsy was sent to the Institution's Pathology Department for routine hematoxylin and eosin evaluation. The remaining part of the biopsy specimen was immediately frozen at $-70^{\circ} \mathrm{C}$ for biological analyses. Samples were taken from patients participating in a single-arm interventional trial conducted in on patients with cervical cancer (ClinicalTrials.gov Identifier: NCT00404326). The clinical results of the trial are published elsewhere (13). Briefly, after signing informed consent, FIGO stage IIIB cancer patients were typed for acetylator phenotype and then treated with hydralazine at $182 \mathrm{mg}$ for rapid-, or $83 \mathrm{mg}$ for slow acetylators, and magnesium valproate at $30 \mathrm{mg} / \mathrm{kg}$, starting from day 7 until chemoradiation ended (external beam radiation $50 \mathrm{~Gy}$ in 2 Gy fractions concurrent with weekly cisplatin at $40 \mathrm{mg} / \mathrm{m}^{2}$, followed by intracavitary therapy to bring dose to point A of at least $85 \mathrm{~Gy})$.

Nucleic acid extraction. RNA from tumors was obtained using the TriReagent RNA extraction kit (Gibco-BRL, Grand Island, NY, USA) following manufacturer's instructions.

Microarray analysis. Target preparation, hybridization, posthybridization, processing, scanning, and normalization were conducted essentially as described (12). Statistical analysis of microarray data was carried out as follows: $\log ^{2}$ gene expression ratios were filtered in order to obtain genes with an absolute ratio $>1$. Gene set was analyzed by means of significance analysis of microarrays (SAM), then obtaining statistical altered genes with a false discovery rate (FDR) $<5 \%$. Significant genes were loaded onto WebGestalt (14), which has the potential to organize and visualize gene sets within the context of Kyoto Encyclopedia of Genes and Genomes (KEGG) (http://www.genome.ad.jp/kegg) biochemical pathways.

Expression profiles comparison. We compared the expression profiles derived from a previous report (15) in which a high-throughput transcriptional analysis in cervical cancer (CC) was done against the $\mathrm{HV}$-induced genes found in the present report. Hence, we compared three expression profiles; HV with 964 significant genes; Up-CC with 1004 overexpressed and Down-CC with 1066 down-regulated genes. Then, ratios were normalized against the absolute media of all expression profiles; obtaining a normalized profile of three different biological entities: normal cervix (represented by absolute value of Down-CC expression ratios) cervical cancer (represented by Up-CC) and cervical cancer treated with epigenetic drugs (represented by HV). In order to look for differences in three profiles described above an initial hierarchical cluster analysis was performed using Spearman rank correlation as similarity metrics and average linkage as the clustering method. To search for differences in biological processes at gene ontology (GO) terms in three expression profiles (HV, Up-CC and Down-CC) we employed FatiGO data mining software, that is an open-access web based program to find differential distributions of biological terms between different groups of genes (16). Retrieved biological processes were taken into account only if adjusted p-values were $<0.01$. Finally, significantly GO biological processes associated terms were graphed.

$C p G$ islands content. We sought for occurrence of $\mathrm{CpG}$ islands in genes shared in Down-CC and HV. A $1 \mathrm{kB}$ upstream sequence of transcriptional start site for each gene was submitted to Genome Table Browser (http://genome.ucsc.edu/ cgi-bin/hgTables) and CpG islands were searched according to definition from Gardiner-Garden and Frommer (17). Accordingly, a $\mathrm{CpG}$ island is defined as a DNA region at least 200 bases long (in our case we used 1000 bp) with a $\mathrm{G}+\mathrm{C}$ content $>50 \%$ and an observed/expected $\mathrm{CpG}$ ratio $\geq 0.6$.

RT-PCR. Primers for NDUFA13 comprised the following: forward 5'aatgcaagaaccaaggcgagtcac3' and reverse 5'aggcat gtcctgcttcacctttga3'. Annealing temperature was $60^{\circ} \mathrm{C}$ for 35 cycles. Products were electrophoresed and visualized under ultraviolet light.

Immunohistochemistry of cervical cancer biopsies. Basal and post-treatment (biopsy at day 8 , before starting chemoradiation) biopsies were analyzed by immunohistochemistry for NDUFA13 and acetylated p53 as follows: 4- $\mu$ m sections of triplicate tissue microarray (pre- and post-treatment with HV) blocks were cut and transferred to adhesive-coated slides. Sections were dewaxed in xylene, rehydrated in graded ethanol, washed in water followed by phosphate-buffered saline (PBS) (0.01 $\mathrm{M} \mathrm{NaH}_{2} \mathrm{PO}_{4} \mathrm{H}_{2} \mathrm{O}, 0.01 \mathrm{M} \mathrm{Na}_{2} \mathrm{HPO}_{4}, 0.15 \mathrm{M}$ $\mathrm{NaCl}, \mathrm{pH}$ 7.2), and blocked for endogenous peroxidase (3\% $\mathrm{H}_{2} \mathrm{O}_{2}$ in $\mathrm{PBS}$ ) for $5 \mathrm{~min}$ at room temperature. Antigen retrieval was performed by pressure cooking for $10 \mathrm{~min}$ in $0.01 \mathrm{mM}$ of sodium citrate $\mathrm{pH}$ 6. Non-specific binding was blocked by incubating the sections in a solution of $10 \%$ FBS diluted in PBS (FBS-PBS) for $30 \mathrm{~min}$ at room temperature. Subsequently, the tissue sections were incubated with the primary antibody [Mouse Anti-Human Complex I (NADH dehydrogenase), $19 \mathrm{kDa}$ subunit GRIM-19 monoclonal antibody, MitoScience LLC] diluted at 1:200 overnight at a temperature of $4^{\circ} \mathrm{C}$ and rabbit anti-human acetyl p53 (Lys320) (Upstate Lake Placid, NY, USA). Signal was detected with the Dako EnVision System, HRP (DAB) (K4011, Dako A/S, Denmark). Tissue sections were counterstained in aqueous hematoxylin, this followed by sequential dehydration using graded ethanols and xylene prior to mounting and cover slipping.

\section{Results}

Gene expression. We evaluated 10 paired tumor samples of cervical cancer patients before and after seven days of HV treatment at a transcriptome level employing Codelink Human Whole Genome Microarrays (each pre-treatment biopsy was hybridized against its post-treatment sample). After gene filtering as described in Patients and methods, we obtained 10,342 genes, which were then analyzed by significance analysis of microarrays (SAM), obtaining 964 signi- 
Table I. Pathways with highest number of up-regulated genes.

\begin{tabular}{lc}
\hline KEGG pathway & No. of genes \\
\hline Ribosome proteins & 38 \\
Oxidative phosphorylation & 34 \\
MAPK signaling pathway & 20 \\
Tight junction & 15 \\
Adherens junction & 14 \\
Regulation of actin cytoskeleton & 14 \\
Pancreatic cancer & 13 \\
Cell cycle & 12 \\
Focal adhesion & 12 \\
LTM & 11 \\
Chronic myeloid leukemia & 10 \\
Insulin signaling pathway & 10 \\
Purine metabolism & 9 \\
Toll-like receptor signaling pathway & 9 \\
Proteasome & 8 \\
Wnt signaling pathway & 8 \\
Antigen processing and presentation & 8 \\
PI3K & 8 \\
Glioma & 8 \\
CCRI & 7 \\
Calcium signaling pathway & 7 \\
TGF-ß signaling pathway & 7 \\
Uboptosis & 8 itin-mediated proteolysis \\
\hline
\end{tabular}

LTM, leukocyte transendothelial migration; PI3K, phosphatidylinositol signaling system; CCRI, cytokine-cytokine receptor interaction; KEGG, Kyoto Encyclopedia of Genes and Genomes.

ficant up-regulated genes with a FDR $<5 \%$ and none downregulated at this threshold. This gene list was uploaded onto WebGestalt, to retrieve its KEGG-associated biochemical pathways (Table I). The two pathways possessing the highest number of up-regulated genes comprised the ribosome protein (RP) and the oxidative phosphorylation (OXPHOS) pathways (Tables II and III), followed by the following cancerrelated pathways: mitogen activated protein kinase (MAPK) signaling, tight junction, adherens junction, actin cytoskeleton, cell cycle, focal adhesion, apoptosis, proteasome, Wnt signaling, and antigen processing and -presentation pathways, among others. The full list of these genes can be found at: http://www.ncbi.nlm.nih.gov/geo/info/linking.html (GEO Submissions GSE8604).

Epigenetic silencing of genes by DNA methylation and histone deacetylation participates in the molecular pathogenesis of the malignant state, therefore, it can be hypothesized that silenced genes in primary cervical cancer can be re-expressed by epigenetic therapy and therefore, the resulting expression profile of the epigenetically treated tumors would group with those found down-regulated in
Table II. Genes from the ribosomal protein pathway.

\begin{tabular}{|c|c|}
\hline Name & Fold-expression \\
\hline RPL10A & 3.0 \\
\hline RPL11 & 3.0 \\
\hline RPL13 & 2.8 \\
\hline RPL13A & 2.6 \\
\hline RPL17 & 4.0 \\
\hline RPL23A & 3.7 \\
\hline RPL24 & 4.2 \\
\hline RPL24 & 2.6 \\
\hline RPL26 & 3.2 \\
\hline RPL26L1 & 2.9 \\
\hline RPL27 & 3.6 \\
\hline RPL27A & 4.4 \\
\hline RPL31 & 4.6 \\
\hline RPL32 & 3.0 \\
\hline RPL34 & 4.0 \\
\hline RPL35 & 3.6 \\
\hline RPL35A & 4.6 \\
\hline RPL36 & 2.7 \\
\hline RPL36A & 4.5 \\
\hline RPL36AL & 2.7 \\
\hline RPL37A & 3.6 \\
\hline RPL38 & 2.5 \\
\hline RPL39 & 3.1 \\
\hline RPL4 & 2.7 \\
\hline RPL41 & 4.7 \\
\hline RPL7A & 5.0 \\
\hline RPLP1 & 2.8 \\
\hline RPS11 & 2.7 \\
\hline RPS12 & 2.6 \\
\hline RPS14 & 3.3 \\
\hline RPS15A & 3.0 \\
\hline RPS16 & 3.1 \\
\hline RPS16 & 2.7 \\
\hline RPS2 & 2.6 \\
\hline RPS27 & 4.5 \\
\hline RPS27A & 2.5 \\
\hline RPS7 & 2.8 \\
\hline UBA52 & 3.1 \\
\hline
\end{tabular}

untreated tumors. To probe our hypothesis, we decided to compare the expression profile we obtained against the expression profile obtained from an unrelated microarray study performed in a similar population of untreated cervical cancer patients (15). Thus, we evaluated them by hierarchical clustering as three different expression profiles: the profile from this study (HV), the up-regulated (Up-CC) and the downregulated (Down-CC) in a previous report (15). This initial comparison indicated that there is clustering between $\mathrm{HV}$ and 
Table III. Genes from the oxidative phosphorylation pathway.

\begin{tabular}{|c|c|}
\hline Name & Fold-expression \\
\hline COX7A2 & 6.9 \\
\hline ATP5C1 & 2.7 \\
\hline ATP5E & 3.0 \\
\hline ATP5G3 & 3.3 \\
\hline ATP5J2 & 3.2 \\
\hline ATP5L & 2.9 \\
\hline COX5A & 3.0 \\
\hline COX5B & 3.7 \\
\hline COX6A1 & 2.7 \\
\hline COX7B & 2.5 \\
\hline $\mathrm{COX} 7 \mathrm{C}$ & 3.5 \\
\hline NDUFA1 & 3.3 \\
\hline NDUFA10 & 2.6 \\
\hline NDUFA2 & 3.0 \\
\hline NDUFA7 & 3.2 \\
\hline NDUFB1 & 3.8 \\
\hline NDUFB2 & 3.5 \\
\hline NDUFB4 & 2.5 \\
\hline NDUFB9 & 2.5 \\
\hline NDUFC1 & 2.5 \\
\hline NDUFS5 & 2.8 \\
\hline NDUFS6 & 2.5 \\
\hline NDUFS8 & 2.8 \\
\hline NDUFV2 & 2.9 \\
\hline PPA2 & 3.2 \\
\hline UQCRB & 2.7 \\
\hline UQCRFS1 & 2.9 \\
\hline UQCRH & 4.4 \\
\hline UQCRQ & 3.6 \\
\hline NDUFA13 & 2.5 \\
\hline NDUFA12 & 2.6 \\
\hline ATP6V1E1 & 2.9 \\
\hline ATP6V1G1 & 2.7 \\
\hline ATP6V0E1 & 5.3 \\
\hline
\end{tabular}

the Down-CC profiles. Fig. 1 shows the extent of coincident individual genes shared in these two sets which are listed in Table IV. Further, the expression profiles of these three set of genes were analyzed in terms of biological processes at gene ontology (GO) entities which were considered for our analysis only if adjusted $p$-values were $<0.01$. Fig. 2 shows the number of genes associated to biological processes, Down-CC are more alike with HV than those intrinsically up-regulated in primary tumors.

Validation by quantitative RT-PCR. NDUFA13 was upregulated, hence, its level of expression, was evaluated by
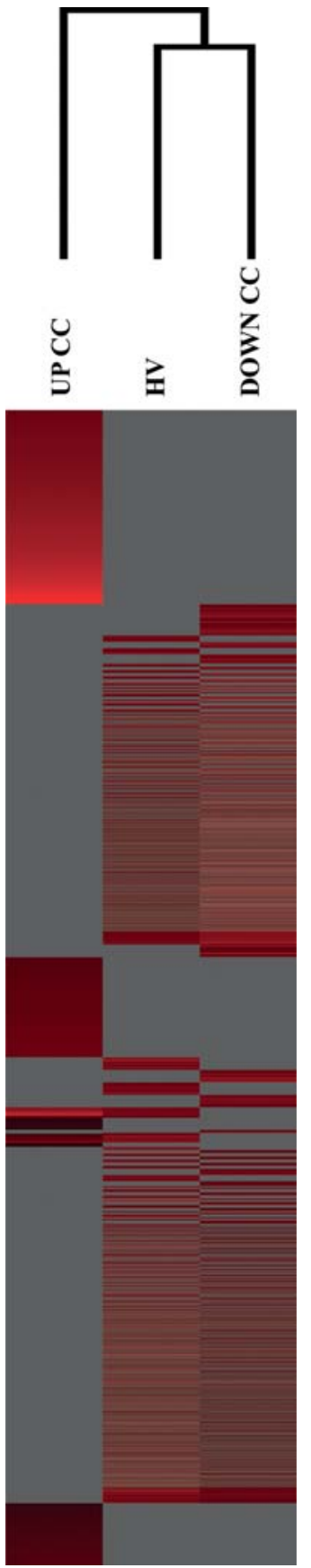

Figure 1. Clustering analysis of the three expression profiles: up-regulated by $\mathrm{HV}$ (this study), up-regulated in cervical cancer (Up-CC) and downregulated in cervical cancer (Down-CC) from ref. 27. There is a major homology between $\mathrm{HV}$ and Down-CC profiles and cluster together.

RT-PCR and immunohistochemistry. There was a tight correlation between the microarray data of this gene with its protein level as with the RNA expression of this gene (Fig. 3a), as well as with its protein as evaluated by immunohistochemistry in the four cases analyzed. A representative case is shown in Fig. 3b. Furthermore, it has been previously demonstrated that HDAC inhibitors not only exert a transcriptional effect, but they are also able to acetylate a number of proteins such as p53; hence, we performed an immunohistochemistry analysis in four patients. Results indicated that all four cases analyzed had increased acetylated p53 at lysine 320. Fig. 4 depicts a representative case. 


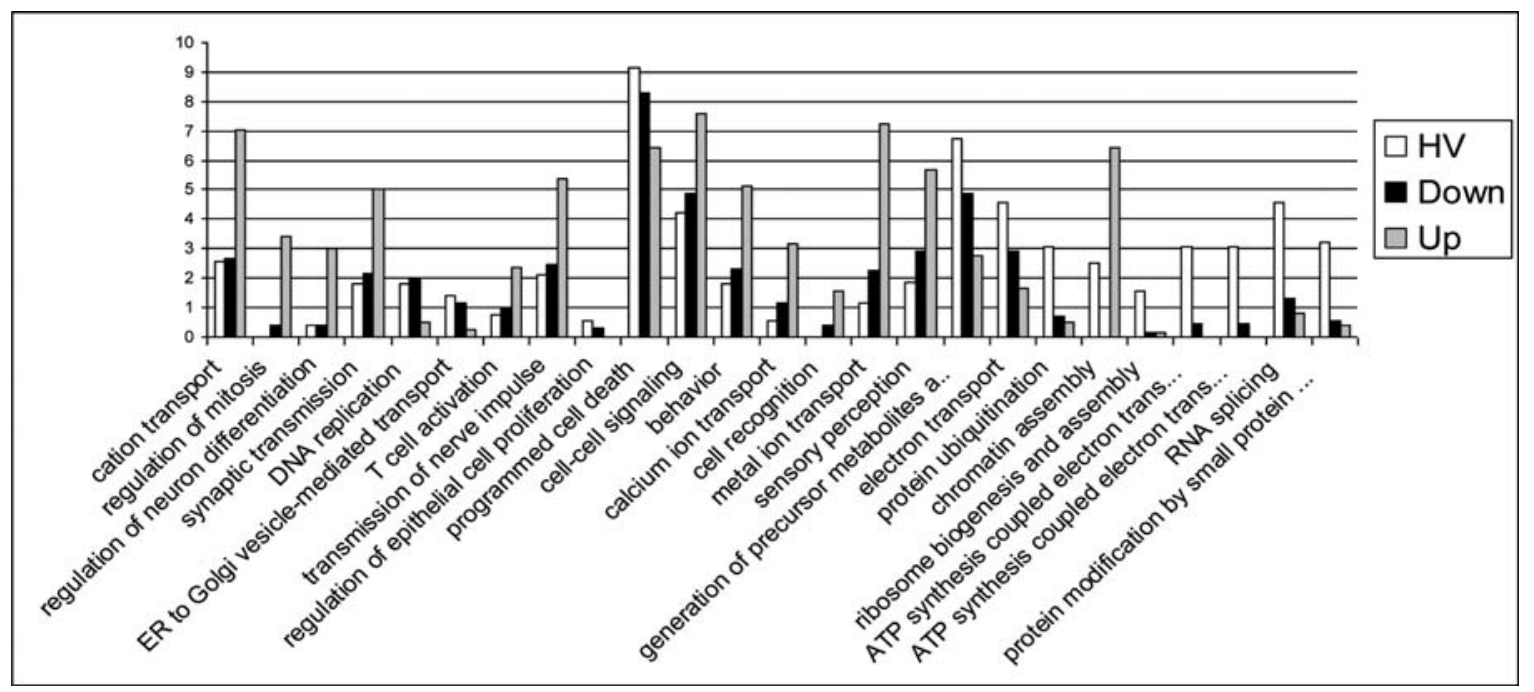

Figure 2. Number of genes associated to specific biological processes in the three expression profiles.

a
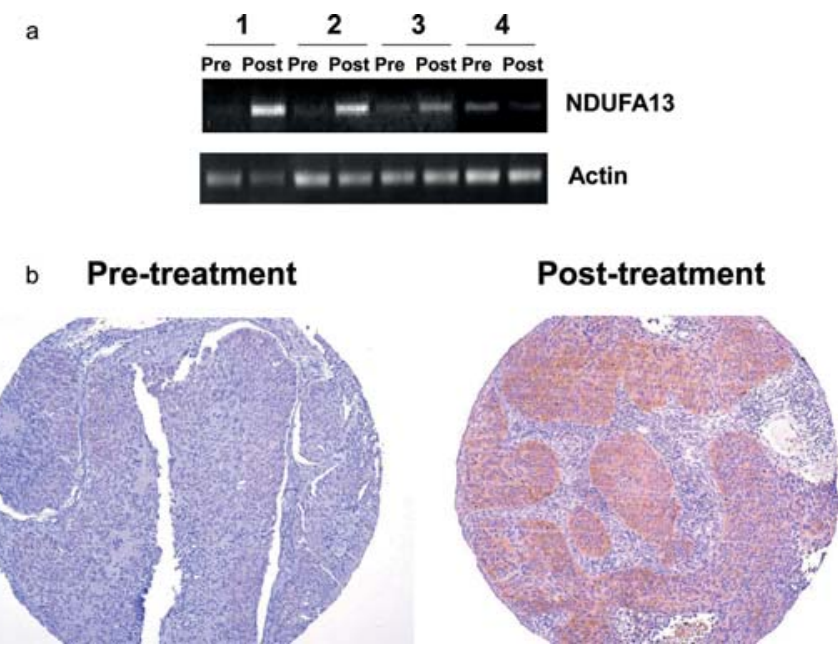

Figure 3. (a) Representative cases of RT-PCR showing changes in expression of NDUFA13 (grim-19) gene. The expression ratio expression analysis in the tumor at lane 4 was zero. (b) Representative case of a tumor before and after HV stained for NDUFA13 (grim-19) protein showing a clear increase in the immunostaining.

\section{Discussion}

The rationale for utilizing epigenetic drugs (DNA methylation and HDAC inhibitors) for cancer treatment has relied on the thought that reversing epigenetic aberrations would turn-on tumor suppressor genes and consequently exert antitumor effects. Most of this knowledge has been generated at the level of individual genes by candidate-gene approach, however, it has been proposed that an epigenomic reactivation screening strategy that combines treatment of cancer cells in vitro with DNA methyltransferase and/or histone deacetylase inhibitors, followed by global gene expression analysis using microarrays to identify up-regulated genes seems to be most effective when complemented by microarray analyses to identify genes repressed in primary tumors. Epigenomic reactivation screening has a number of key advantages
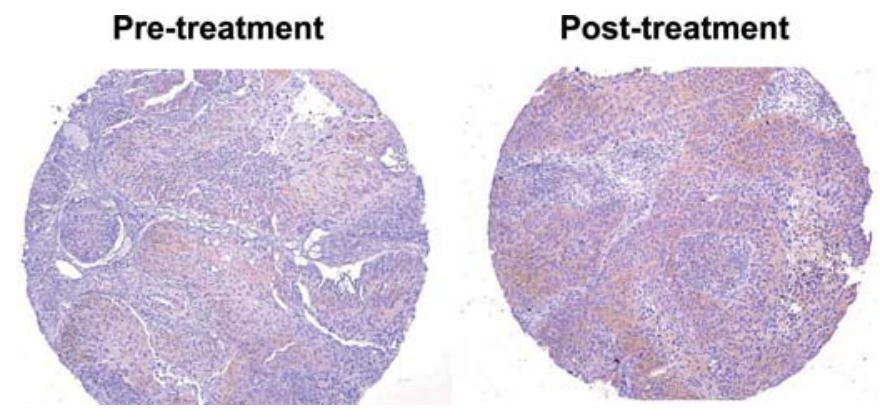

Figure 4. Representative case of a tumor before and after HV stained with a p53 antibody against acetylated lysine 320 showing increased staining.

over direct analysis of cancer-associated DNA methylation changes; most notably, it directly identifies genes in which epigenetic changes lead to altered gene expression. Nevertheless, this kind of analyses has only been performed in cell lines. For solid tumors, the only information regarding the effects on the cancer transcriptome of $\mathrm{HV}$ comes from our study in breast cancer, however, the information provided there is limited as only a single pair of pre and post-treatment sample could be analyzed (12).

Among 10,226 genes that underwent 2-fold over or underexpression, 964 were significantly overexpressed. Functional analysis of these genes underscore that the two pathways that had the greatest number of genes up-regulated were genes coding for ribosomal proteins (38 genes) and oxidative phosphorylation pathway (34 genes). This result is in agreement with our previous study in breast cancer tumors analyzed in a similar manner (12).

Ribosomal proteins are integral components of the basal cellular machinery involved in protein synthesis. Various individual ribosomal proteins and also translation, initiation, and elongation factors have been found to play roles in regulating cell growth, transformation, and death, giving rise to increasing speculation that components of the translational apparatus can act as multifunctional proteins $(18,19)$. The most 
Table IV. Genes up-regulated by hydralazine-valproate and found down-regulated in cervical cancer patients.

\begin{tabular}{|c|c|}
\hline Symbol & Name \\
\hline ACCN4 & Amiloride-sensitive cation channel 4, pituitary \\
\hline ACTN4 & Actinin, $\alpha 4$ \\
\hline AIG1 & Androgen-induced 1 \\
\hline AKAP13 & In multiple clusters \\
\hline APP & Amyloid B (A4) precursor protein (peptidase nexin-II, Alzheimer disease) \\
\hline ARHGAP10 & Rho GTPase activating protein 10 \\
\hline BCAP29 & B-cell receptor-associated protein 29 \\
\hline BTF3 & Basic transcription factor 3 \\
\hline CAPNS2 & Calpain, small subunit 2 \\
\hline CD59 & In multiple clusters \\
\hline CSTA & Cystatin A (stefin A) \\
\hline CSTB & Cystatin B (stefin B) \\
\hline DSTN & In multiple clusters \\
\hline EBPL & Emopamil binding protein-like \\
\hline EEF1A1 & In multiple clusters \\
\hline EEF2 & Eukaryotic translation elongation factor 2 \\
\hline EGFR & Epidermal growth factor receptor (v-erb-b) oncogene homolog, avian) \\
\hline ELOVL5 & ELOVL family member 5, elongation of long chain fatty acids (FEN1/Elo2, SUR4/Elo3-like) \\
\hline ETS2 & V-ets erythroblastosis virus E26 oncogene homolog 2 (avian) \\
\hline FOXO3A & Data not found \\
\hline FUNDC2 & FUN14 domain containing 2 \\
\hline GADD45B & Growth arrest and DNA-damage-inducible, $\beta$ \\
\hline GDI2 & GDP dissociation inhibitor 2 \\
\hline GPR153 & G protein-coupled receptor 153 \\
\hline HEBP1 & Heme binding protein 1 \\
\hline HIST1H3C & Histone cluster $1, \mathrm{H} 3 \mathrm{c}$ \\
\hline KCNAB1 & In multiple clusters \\
\hline KCNMA1 & Potassium large conductance calcium-activated channel, subfamily $M, \alpha$ member 1 \\
\hline KRT5 & Data not found \\
\hline MBNL1 & Muscleblind-like (Drosophila) \\
\hline MEIS1 & In multiple clusters \\
\hline MRPL33 & Mitochondrial ribosomal protein L33 \\
\hline MYL6 & Myosin, light chain 6, alkali, smooth muscle and non-muscle \\
\hline NCOA5 & Nuclear receptor coactivator 5 \\
\hline NEK7 & NIMA (never in mitosis gene a)-related kinase 7 \\
\hline NES & Nestin \\
\hline PBX1 & Pre-B-cell leukemia homeobox 1 \\
\hline PLEKHA5 & Pleckstrin homology domain containing, family A member 5 \\
\hline PLSCR1 & Phospholipid scramblase 1 \\
\hline PTEN & Phosphatase and tensin homolog (mutated in multiple advanced cancers 1) \\
\hline PTPRA & Protein tyrosine phosphatase, receptor type, A \\
\hline RBMS1 & RNA binding motif, single stranded interacting protein 1 \\
\hline RPL10A & In multiple clusters \\
\hline RPL13A & Ribosomal protein L13a \\
\hline RPL36A & Ribosomal protein L36a \\
\hline RPL4 & In multiple clusters \\
\hline RPS2 & In multiple clusters \\
\hline S100A4 & S100 calcium binding protein A4 \\
\hline S100A9 & S100 calcium binding protein A9 \\
\hline SBDS & Shwachman-Bodian-Diamond syndrome \\
\hline SERP1 & Stress-associated endoplasmic reticulum protein 1 \\
\hline SHC1 & SHC (Src homology 2 domain containing) transforming protein 1 \\
\hline
\end{tabular}


Table IV. Continued.

\begin{tabular}{ll}
\hline Symbol & \\
\hline SIL1 & SIL1 homolog, endoplasmic reticulum chaperone (S. cerevisiae) \\
SMAD5 & SMAD family member 5 \\
SNTB2 & Syntrophin, $\mathbf{3} 2$ (dystrophin-associated protein A1, 59 kDa, basic component 2) \\
STX17 & Syntaxin 17 \\
SUV420H1 & Suppressor of variegation 4-20 homolog 1 (Drosophila) \\
TCF4 & Transcription factor 4 \\
TPD52L1 & Tumor protein D52-like 1 \\
TPM1 & Tropomyosin 1 $(\alpha)$ \\
TUBB6 & Tubulin, $\beta 6$ \\
UQCRFS1 6 \\
VAPA & Ubiquinol-cytochrome c reductase, Rieske iron-sulfur polypeptide 1 \\
ZBTB16 & VAMP (vesicle-associated membrane protein)-associated protein A, 33 kDa \\
ZFP36 & In multiple clusters \\
ZNF185 & In multiple clusters \\
ZNF24 & Zinc finger protein 185 (LIM domain) \\
\hline
\end{tabular}

Genes in bold have CpG islands in their promoters. Down-regulated in ref. 15.

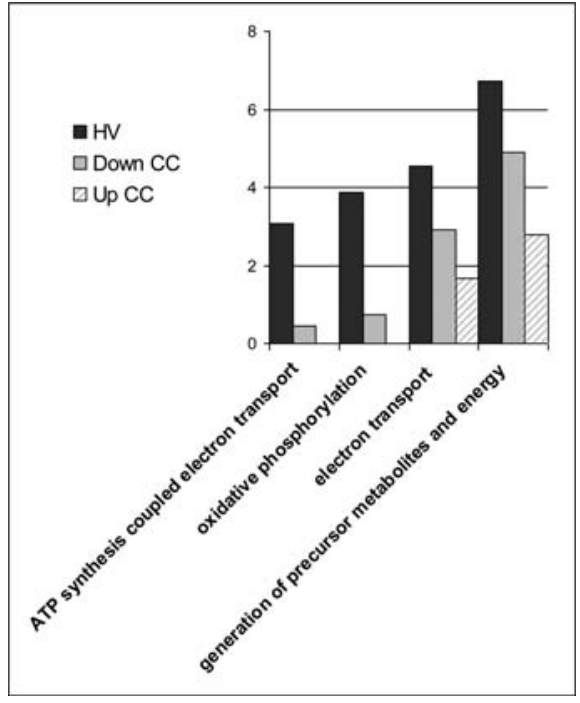

Figure 5. Pathways related to energy production in terms of gene ontology. HV treatment up-regulates a higher number of genes participating in ATP synthesis, coupled electron transport, oxidative phosphorylation, electron transport and generation of precursor metabolites and energy.

compelling suggestion concerning the ribosomal protein role in cancer derives from a study in which several hundred lines of zebra fish (Danio rerio), each heterozygous for a recessive embryonic lethal mutation, were generated and screened for lines that displayed early mortality and/or gross evidence of tumors. The authors identified 12 lines with elevated cancer incidence; surprisingly, 11 of the 12 lines were each heterozygous for a mutation in a different ribosomal protein (RP) gene. These data led authors to suggest that many RP genes may act as haplo-insufficient tumor suppressors (20).
Interestingly, nine of these 11 genes (S7, S8, S15a, L7, L13, $L 23 a, L 35, L 36$, and L36a) were up-regulated in cervical cancer tumors, as shown in Table II. Further, in humans there is a possible association of mutations in one particular RP gene with cancer: approximately $25 \%$ of both sporadic and familial cases of Diamond-Blackfan anemia (DBA) are associated with a $\operatorname{rpS} 19$ mutation (21), and this syndrome includes an increased risk of developing malignancies $(22,23)$. Recent data demonstrate that in addition, RPS19 deficiency leads to co-down-regulation of multiple ribosomal protein genes, as well as down-regulation of the genes involved in transcription, translation, and expression changes for multiple cancer-related genes, which suggests a molecular basis for increased risk for malignancy in these patients (24). Thus, the current findings in cervical cancer patients treated with epigenetic drugs clearly indicate the need for analyzing the role of RP in the molecular pathogenesis of this malignancy.

The OXPHOS system consists of a respiratory chain, the adenosine triphosphate (ATP) synthase, and several mitochondrial-membrane metabolite transporters. The respiratory chain is organized in four multiprotein complexes, as well as the ATP synthase. Individual subunits are encoded by both the mitochondrial and the nuclear genome. Defects in the OXPHOS system result in devastating diseases, and in recent years its role in cancer has begun to be studied (25). For example, the gene codifying the nicotinamide adenine dinucleotide (NADH) dehydrogenase (ubiquinone) $1 \alpha$ subcomplex 13 is known to be down-regulated in basal cell carcinomas (26). This gene, also denominated NDUFA13 or GRIM19, was initially identified as a pro-apoptotic gene mediating retinoid antitumor effects and indispensable for proper assembling and functioning of complex I of respiratory chain (27); its down-regulation has also been found in doxorubicin-resistant A431 cells (28). Further, recent studies 
have pointed out the importance of the OXPHOS pathway in carcinogenesis and resistance to cancer therapy $(28,29)$, and it has been suggested that loss of cell dependence on oxidative metabolism is an important factor in tumor development. In this line, it has been reported that drug-resistant tumors exhibit low oxidative phosphorylation and high glycolysis (30). Furthermore, it has been demonstrated that oxidative phosphorylation, and possibly increased oxidative phosphorylation, plays a crucial role in Bax and Bak activation and cell death (31). A further analysis of metabolic pathways related to energy production in terms of gene ontology (Fig. 5) remarks on the fact that $\mathrm{HV}$ treatment up-regulates a higher number of genes participating in ATP synthesis, coupled electron transport, oxidative phosphorylation, electron transport and generation of precursor metabolites and energy, as compared to normal cervix and cervical carcinoma, in fact, the two first classes of genes are absent in cervical cancers suggesting the malignant cells tend to have a decreased 'energetical' function which could help tumors to evade apoptosis. Herein, we demonstrated that the epigenetic drugs tested are able to up-regulate a significant number of genes belonging to this pathway suggesting that hydralazine and valproate change tumor metabolism from anaerobic glycolysis to oxidative phosphorylation, which could restore the cells' sensitivity to programmed cell death. Nevertheless, this statement is only hypothetical and needs to be further studied.

Currently, there is no information on whole genome transcriptional changes induced in solid tumors by epigenetic agents; however, we have previously compared the pathways in regard to the number of genes up-regulated in primary colon primary cancers resistant to therapy against our previous data in the colon carcinoma cell line SW480 treated with HV. As reported (32) we found a mirror image on the expressed genes in each of the pathways analyzed suggesting that epigenetic agents can revert the transcriptional signature associated with chemoresistance. Current data on transcriptional changes induced in cancer cell lines by epigenetic agents indicate that not all up-regulated genes have $\mathrm{CpG}$ islands in their promoters and theoretically silenced by epigenetic mechanisms, but that gene up-regulation in a proportion of them is secondary of pathway activation. As shown in Table IV, $40 \%$ of up-regulated genes by HV coincident with the downregulated in primary cervical tumors have $\mathrm{CpG}$ islands.

Finally, current evidence suggests that the antitumor effects of HDAC inhibitors depend not only on transcriptioninduced changes in cancer-related genes, but also on the acetylation of transcription factors and other proteins (33). We also show here, that as observed in cell lines $(34,49)$, this indeed does occur in the clinical setting, as we found an increase in p53 immunostaining with an antibody against the acetylated 320 lysine of p53. Acetylation at this residue is needed for the HDAC inhibitor-induced apoptotic effect (35).

In summary, the fact that the overall effects of valproate and other HDAC inhibitors alone or with DNA methylation inhibitors on cells can be the induction of cell differentiation, arrest of proliferation, apoptosis, antiangiogenesis, anti-metastasis or reversion of resistance argues towards a mechanism involving changes in the expression of hundreds of genes that in turn may disrupt the 'malignant homeostasis'. Such an effect could be potentiated when these agents are administered along with other cytotoxics and radiation. Nevertheless, a detailed analysis of the changes induced by hydralazine and valproate in each of these pathways is mandatory to better understand its antitumor effects and for developing predictive tools for response and treatment individualization.

\section{Acknowledgements}

This study was supported by CONACyT grants SALUD2002-C01-6579, 60517, AVANCE C01-294, and by Psicofarma, S.A. de C.V., Mexico. Sponsors did not participate in study design, collection, analysis, and interpretation of data, writing of the study, nor decision to submit it for publication.

\section{References}

1. Hellebrekers DM, Griffioen AW and van Engeland M: Dual targeting of epigenetic therapy in cancer. Biochim Biophys Acta 1775: 76-91, 2007.

2. Catalano MG, Fortunati N, Pugliese M, Poli R, Bosco O, Mastrocola R, Aragno M and Boccuzzi G: Valproic acid, a histone deacetylase inhibitor, enhances sensitivity to doxorubicin in anaplastic thyroid cancer cells. J Endocrinol 191: 465-472, 2006.

3. Maggio SC, Rosato RR, Kramer LB, Dai Y, Rahmani M, Paik DS, Czarnik AC, Payne SG, Spiegel S and Grant S: The histone deacetylase inhibitor MS-275 interacts synergistically with fludarabine to induce apoptosis in human leukemia cells. Cancer Res 64: 2590-2600, 2004.

4. Yeow WS, Ziauddin MF, Maxhimer JB, Shamimi-Noori S, Baras A, Chua A, Schrump DS and Nguyen DM: Potentiation of the anticancer effect of valproic acid, an antiepileptic agent with histone deacetylase inhibitory activity, by the kinase inhibitor Staurosporine or its clinically relevant analogue UCN-01. Br J Cancer 94: 1436-1445, 2006.

5. Chávez-Blanco A, Pérez-Plasencia C, Pérez-Cárdenas E, Carrasco-Legleu C, Rangel-Lopez E, Segura-Pacheco B, TajaChayeb L, Trejo-Becerril C, Gonzalez-Fierro A, Candelaria M, Cabrera G and Duenas-Gonzalez A: Antineoplastic effects of the DNA methylation inhibitor hydralazine and the histone deacetylase inhibitor valproic acid in cancer cell lines. Cancer Cell Int 6: 2, 2006.

6. Forrer C, Hofstetter B and Pruschy M: Treatment with demethylating agent 5-Aza-2'-Deoxy-Cytidine (DAC) leads to enhanced sensitivity to ionizing radiation (IR) in colorectal carcinoma. Radiat Oncol 73: S1-S497, 2004.

7. Dote H, Cerna D, Burgan WE, Carter DJ, Cerra MA, Hollingshead MG, Camphausen K and Tofilon PJ: Enhancement of in vitro and in vivo tumor cell radiosensitivity by the DNA methylation inhibitor zebularine. Clin Cancer Res 11: 4571-4579, 2005.

8. Camphausen K, Cerna D, Scott T, Sproull M, Burgan WE, Cerra MA, Fine $\mathrm{H}$ and Tofilon PJ: Enhancement of in vitro and in vivo tumor cell radiosensitivity by valproic acid. Int $\mathrm{J}$ Cancer 114: 380-386, 2005.

9. Karpf AR: Epigenomic reactivation screening to identify genes silenced by DNA hypermethylation in human cancer. Curr Opin Mol Ther 9: 231-241, 2007.

10. Shi H, Wei SH, Leu YW, Rahmatpanah F, Liu JC, Yan PS, Nephew KP and Huang TH: Triple analysis of the cancer epigenome: an integrated microarray system for assessing gene expression, DNA methylation, and histone acetylation. Cancer Res 63: 2164-2171, 2003.

11. Li L, Shi H, Yiannoutsos C, Huang TH and Nephew KP: Epigenetic hypothesis tests for methylation and acetylation in a triple microarray system. J Comput Biol 12: 370-390, 2005.

12. Arce C, Pérez-Plasencia C, González-Fierro A, de la CruzHernandez E, Revilla-Vazquez A, Chavez-Blanco A, TrejoBecerril C, Perez-Cardenas E, Taja-Chayeb L, Bargallo E, Villarreal P, Ramirez T, Vela T, Candelaria M, Camargo MF, Robles E and Duenas-Gonzalez A: A proof-of-principle study of epigenetic therapy added to neoadjuvant doxorubicin cyclophosphamide for locally advanced breast cancer. PLoS One 1: e98, 2006. 
13. Candelaria M, Cetina L, Coronel J, Cantu D, Rivera L, PerezCardenas E, de la Cruz-Hernández E, Gonzalez-Fierro A, Chavez-Blanco A, Trejo-Becerril C, Taja-Chayeb L, Chanona J and Duenas-Gonzalez A: Epigenetic therapy added to cisplatin chemoradiation in FIGO stage IIIB cervical cancer. Eur J Gynecol Oncol 31: 386-391, 2010.

14. Zhang B, Kirov S and Snoddy J: WebGestalt: an integrated system for exploring gene sets in various biological contexts. Nucleic Acids Res 33: W741-W748, 2005.

15. Perez-Plasencia C, Vazquez-Ortiz G, Lopez-Romero R, PinaSanchez P, Moreno J and Salcedo M: Genome wide expression analysis in HPV16 cervical cancer: identification of altered metabolic pathways. Infect Agent Cancer 2: 16, 2007.

16. Al-Shahrour F, Minguez P, Vaquerizas JM, Conde L and Dopazo J: Babelomics: a suite of web-tools for functional annotation and analysis of group of genes in high-throughput experiments. Nucleic Acids Res 33: W460-W464, 2005.

17. Gardiner-Garden $\mathrm{M}$ and Frommer $\mathrm{M}$ : $\mathrm{CpG}$ islands in vertebrate genomes. J Mol Biol 196: 261-282, 1987.

18. Rosenwald IB: The role of translation in neoplastic transformation from a pathologist's point of view. Oncogene 23: 3230-3247, 2004.

19. Ruggero D and Pandolfi PP: Does the ribosome translate cancer? Nat Rev Cancer 3: 179-192, 2003.

20. Amsterdam A, Sadler KC, Lai K, Farrington S, Bronson RT, Lees JA and Hopkins N: Many ribosomal protein genes are cancer genes in zebrafish. PLoS Biol 2: E139, 2004.

21. Draptchinskaia N, Gustavsson P, Andersson B, Pettersson M, Willig TN, Dianzani I, Ball S, Tchernia G, Klar J, Matsson H, Tentler D, Mohandas N, Carlsson B and Dahl N: The gene encoding ribosomal protein S19 is mutated in Diamond-Blackfan anemia. Nat Genet 21: 169-175, 1999

22. Wasser JS, Yolken R, Miller DR and Diamond L: Congenital hypoplastic anemia (Diamond-Blackfan syndrome) terminating in acute myelogenous leukemia. Blood 51: 991-995, 1978.

23. Lipton JM, Federman N, Khabbaze Y, Schwartz CL, Hilliard LM, Clark JI, Vlachos A; Diamond-Black Anemia Registry: Diamond-Black Anemia Registry: Osteogenic sarcoma associated with Diamond-Blackfan anemia: a report from the Diamond-Blackfan Anemia Registry. J Pediatr Hematol Oncol 23: 39-44, 2001.

24. Gazda HT, Kho AT, Sanoudou D, Zaucha JM, Kohane IS, Sieff CA and Beggs AH: Defective ribosomal protein gene expression alters transcription, translation, apoptosis, and oncogenic pathways in Diamond-Blackfan anemia. Stem Cells 24: 2034-2044, 2006.

25. Smeitink J, van den Heuvel L and DiMauro S: The genetics and pathology of oxidative phosphorylation. Nat Rev Genet 5: $342-352,2001$.
26. Mamelak AJ, Kowalski J, Murphy K, Yadava N, Zahurak M, Kouba DJ, Howell BG, Tzu J, Cummins DL, Liegeois NJ, Berg K and Sauder DN: Downregulation of NDUFA1 and other oxidative phosphorylation-related genes is a consistent feature of basal cell carcinoma. Exp Dermatol 14: 336-348, 2005.

27. Huang G, Lu H, Hao A, Ng DC, Ponniah S, Guo K, Lufei C, Zeng Q and Cao X: GRIM-19, a cell death regulatory protein, is essential for assembly and function of mitochondrial complex I. Mol Cell Biol 24: 8447-8456, 2004.

28. Wong TW, Yu HY, Kong SK, Fung KP and Kwok TT: The decrease of mitochondrial NADH dehydrogenase and drug induced apoptosis in doxorubicin resistant A431 cells. Life Sci 67: 1111-1118, 2000.

29. Rodríguez-Enriquez S, Vital-González PA, Flores-Rodríguez FL, Marin-Hernandez A, Ruiz-Azuara L and Moreno-Sanchez R: Control of cellular proliferation by modulation of oxidative phosphorylation in human and rodent fast-growing tumor cells. Toxicol Appl Pharmacol 215: 208-217, 2006.

30. Harper ME, Antoniou A, Villalobos-Menuey E, Russo A, Trauger R, Vendemelio M, George A, Bartholomew R, Carlo D, Shaikh A, Kupperman J, Newell EW, Bespalov IA, Wallace SS, Liu Y, Rogers JR, Gibbs GL, Leahy JL, Camley RE, Melamede R and Newell MK: Characterization of a novel metabolic strategy used by drug-resistant tumor cells. FASEB J 16: 1550-1557, 2002.

31. Tomiyama A, Serizawa S, Tachibana K, Sakurada K, Samejima H, Kuchino Y and Kitanaka C: Critical role for mitochondrial oxidative phosphorylation in the activation of tumor suppressors Bax and Bak. J Natl Cancer Inst 98: 1462-1473, 2006.

32. Perez-Plasencia $C$ and Duenas-Gonzalez A: Can the state of cancer chemotherapy resistance be reverted by epigenetic therapy? Mol Cancer 5: 27, 2006

33. Bolden JE, Peart MJ and Johnstone RW: Anticancer activities of histone deacetylase inhibitors. Nat Rev Drug Discov 5: 769-784, 2006.

34. de la Cruz-Hernandez E, Perez-Cardenas E, Contreras-Paredes A, Cantu D, Mohar A, Lizano M and Duenas-Gonzalez A: The effects of DNA methylation and histone deacetylase inhibitors on human papillomavirus early gene expression in cervical cancer, an in vitro and clinical study. Virol J 4: 18, 2007.

35. Terui T, Murakami K, Takimoto R, Takahashi M, Takada K, Murakami T, Minami S, Matsunaga T, Takayama T, Kato J and Niitsu Y: Induction of PIG3 and NOXA through acetylation of p53 at 320 and 373 lysine residues as a mechanism for apoptotic cell death by histone deacetylase inhibitors. Cancer Res 63: 8948-8954, 2003. 